\title{
The Impact of Type 2 Diabetes on the Efficacy of ADP Receptor Blockers in Patients with Acute ST Elevation Myocardial Infarction: A Pilot Prospective Study
}

\author{
Matej Samoš, ${ }^{1}$ Marián Fedor, ${ }^{2}$ František Kovár̆, ${ }^{1}$ \\ Peter Galajda, ${ }^{1}$ Tomáš Bolek, ${ }^{1}$ Lucia Stančiaková, ${ }^{2}$ Jana Fedorová, ${ }^{3}$ \\ Ján Staško, ${ }^{2}$ Peter Kubisz, ${ }^{2}$ and Marián Mokáň ${ }^{1}$ \\ ${ }^{1}$ Department of Internal Medicine I, Jessenius Faculty of Medicine in Martin, Comenius University in Bratislava, \\ 03659 Martin, Slovakia \\ ${ }^{2}$ National Centre of Hemostasis and Thrombosis, Department of Hematology and Blood Transfusion, \\ Jessenius Faculty of Medicine in Martin, Comenius University in Bratislava, 03659 Martin, Slovakia \\ ${ }^{3}$ HemoMedika, Centre of Thrombosis and Hemostasis, 03601 Martin, Slovakia
}

Correspondence should be addressed to Matej Samoš; matej.samos@gmail.com

Received 23 March 2016; Revised 27 May 2016; Accepted 22 June 2016

Academic Editor: Konstantinos Papatheodorou

Copyright (C) 2016 Matej Samoš et al. This is an open access article distributed under the Creative Commons Attribution License, which permits unrestricted use, distribution, and reproduction in any medium, provided the original work is properly cited.

\begin{abstract}
Background. The aim of this study was to validate the impact of type 2 diabetes (T2D) on the platelet reactivity in patients with acute ST elevation myocardial infarction (STEMI) treated with adenosine diphosphate (ADP) receptor blockers. Methods. A pilot prospective study was performed. Totally 67 patients were enrolled. 21 patients had T2D. Among all study population, 33 patients received clopidogrel and 34 patients received prasugrel. The efficacy of ADP receptor blocker therapy had been tested in two time intervals using light transmission aggregometry with specific inducer and vasodilator-stimulated phosphoprotein phosphorylation (VASP-P) flow cytometry assay. Results. There were no significant differences in platelet aggregability among T2D and nondiabetic (ND) group. The platelet reactivity index of VASP-P did not differ significantly between T2D and ND group $(59.4 \pm 30.9 \%$ versus $60.0 \pm 25.2 \%$ and $33.9 \pm 25.3 \%$ versus $38.6 \pm 29.3 \%$ in second testing). The number of ADP receptor blocker nonresponders did not differ significantly between T2D and ND patients. The time interval from ADP receptor blocker loading dosing to the blood sampling was similar in T2D and ND patients in both examinations. Conclusion. This prospective study did not confirm the higher platelet reactivity and higher prevalence of ADP receptor blocker nonresponders in T2D acute STEMI patients.
\end{abstract}

\section{Background}

Type 2 diabetes (T2D) is a strong and independent risk factor of cardiovascular disease in both men and women [13]. Diabetes not only increases the risk of acute coronary syndrome (ACS) but also increases the mortality associated with this acute event. T2D worsens the course of ACS and increases the risk of its complications including cardiogenic shock and death [4] and remains an independent predictor of worse prognosis of ACS, even after excluding the impact of older age, more severe coronary artery disease, and worse left ventricular function in patients with T2D [5]. Moreover, T2D is connected with platelet dysfunction $[6,7]$, which increases the risk of arterial thrombosis. Several recently published studies and case reports pointed at a failure in antiplatelet response to clopidogrel (the most frequently used ADP receptor blocker in ACS patients) which might be connected with insulin resistance and T2D. High clopidogrel on-treatment platelet reactivity is associated with increased risk of thrombotic adverse events including stent thrombosis [8-10]. The aim of this preliminary prospective study was to validate the impact of $\mathrm{T} 2 \mathrm{D}$ in relation to the on-treatment 
platelet reactivity in patients with acute ST elevation myocardial infarction (STEMI) treated with ADP receptor blocker therapy.

\section{Methods}

2.1. Study Design and Patients. A single centre, pilot prospective observational study in patients with acute STEMI was performed. All patients underwent coronary angiography and primary percutaneous coronary intervention (pPCI) of culprit coronary lesion. Totally 67 consecutive presentations of patients (37 men and 30 women; mean age 67 years; the youngest patient was 34-year-old and the oldest patient was 89-year-old) with acute STEMI and pPCI of coronary lesion were enrolled in this study. Patients with multivessel coronary disease planned for surgical revascularization, patients treated only conservatively, and hemodynamically unstable patients (i.e., patients in Killip class IV) had been excluded from this study. Additionally, patients with hypertensive crisis, kidney, and liver failure had been also excluded from study population. Moreover, patients with medication which could interfere with the action of ADP receptor blockers, such as omeprazole, fluconazole, or morphine were also excluded from this study. All patients should be ADPRB naïve prior to myocardial infarction. Patients with known and correctly diagnosed history of T2D had been assigned to T2D group. This group included 21 patients. In all patients without previous history of T2D the glycated hemoglobin (HbA1C) levels were assessed in order to exclude patients with undiagnosed T2D. Patients with HbA1C levels > 5.7\% DCCT were considered to be patients with possibly undiagnosed T2D and these patients had been excluded from the study. Subsequently, a standard oral glucose tolerance test $(75 \mathrm{~g}$ of glucose was administrated in $100 \mathrm{~mL}$ of water and a venous blood sample was taken two hours after the glucose administration) was performed in the rest of the patients without previous history of T2D one month after the hospital discharge. Patients with blood glucose value $>7.8 \mathrm{mmol} / \mathrm{L}$ two hours after the glucose administration had been also excluded from the study. Patients without previous history of T2D, with HbA1C levels < 5.7\% DCCT and blood glucose value $<7.8 \mathrm{mmol} / \mathrm{L}$ shown in oral glucose tolerance test had been assigned to nondiabetic (ND) group. The basic demographic data and concomitant medication in the study population are shown in Table 1. Among all study population, 33 patients received clopidogrel (a loading dose of $600 \mathrm{mg}$ followed by a maintenance dose of $75 \mathrm{mg} /$ daily) and 34 patients received prasugrel (a loading dose of $60 \mathrm{mg}$ followed by a maintenance dose of $10 \mathrm{mg}$ /daily). Totally 22 patients enrolled in this study were $>75$ years old $(9$ T2D patients and $13 \mathrm{ND}$ patients); all of these patients were clopidogrel-treated. All patients received aspirin therapy (a loading dose of 200-400 mg followed by a maintenance dose of $100 \mathrm{mg} /$ daily) and a weight-adjusted unfractionated heparin therapy $(100 \mathrm{IU} / \mathrm{kg}$ intravenously) prior to $\mathrm{pPCI}$ in order to prevent periprocedural thrombosis. No other antiplatelet or anticoagulant therapy was administrated in studied population. The drug compliance had been carefully monitored with a healthcare professional, who supervised all antiplatelet drug administration. Venous blood samples had been taken after obtaining the written informed content in all patients enrolled in this study in order to assess the ontreatment platelet reactivity using selected platelet function tests.

2.2. Platelet Function Testing. Blood samples had been taken using $3.8 \%$ citrate vacutainer blood collection tubes. Blood samples had been immediately analyzed within first 2 hours from blood sampling. The samples had been taken in the following time intervals:

Sample 1, at the time of patient arrival to cath laboratory: this sample aimed to test the efficacy of ADP receptor blocker given in loading doses prior to the urgent coronary angiography and PPCI of coronary lesion.

Sample 2, one hour after the administration of first ADP receptor blocker maintenance dose: this sample aimed to test the efficacy of in-hospital ADP receptor blocker therapy given in maintenance dosage.

The platelet reactivity was tested using light transmission aggregometry (LTA) with specific inducer (ADP) and vasodilator-stimulated phosphoprotein phosphorylation (VASP-P) flow cytometry assay.

Light Transmission Aggregometry (LTA). this method represents recently the "golden standard" of platelet function testing. ADP $(10 \mu \mathrm{mol} / \mathrm{L})$ was used as specific inducer for ADP receptor blocker efficacy testing. LTA was examined with Chrono-Log model 700 (Chronolog Corporation, Havertown, PA, USA). The platelet aggregability was assessed on the basis of the change in the plasma turbidity after the addition of the specific inducer. Residual platelet aggregability $>50 \%$ after the addition of ADP was considered to be high ontreatment platelet reactivity (HTPR).

VASP-P Flow Cytometry Assay. In this analysis, we used PLT VASP/P2Y12 assay kits (Diagnostica Stago, France). Sample of citrate blood was incubated with prostaglandin E1 (PGE1) and PGE1 + ADP (activated platelets). After cellular permeabilization by nonionic detergent, VASP-P is labeled by indirect no-wash immunofluorescence using a specific monoclonal antibody. Dual color flow cytometry analysis then allowed comparison of the 2 tested conditions. Analysis was carried out on FACSCalibur flow cytometer (BD Biosciences, San Jose, California). In the final step, the platelet reactivity index (PRI) was calculated using corrected mean VASP fluorescence intensities (MFIc) in the presence of PGE1 alone (resting platelets) or PGE1 + ADP simultaneously (activated platelets). Index represented the ratio of activated/resting platelets and was calculated according to the following equation:

$$
\mathrm{PRI}=\frac{\mathrm{MFIc}^{\mathrm{PGE} 1}-\mathrm{MFIc}^{[\mathrm{PGE} 1+\mathrm{ADP}]}}{\text { MFIc }^{\text {PGE1 }}} \times 100 .
$$

The resulting value described PRI to ADP treatment in the range of $0 \%$ to $100 \%$. Values of PRI above $50 \%$ were considered as determinant of HTPR and inadequate response to treatment. 
TABLE 1: Demographic data and concomitant medication in studied acute STEMI patients.

\begin{tabular}{|c|c|c|c|}
\hline & T2D group & ND group & Significance \\
\hline Number of patients (men/women) & $21(11 / 10)$ & $46(26 / 20)$ & NS \\
\hline T2D duration (years) & $11.4 \pm 7.6$ & - & $\mathrm{N} / \mathrm{A}$ \\
\hline HbA1C levels & $7.2 \pm 1.9 \%$ & $4.03 \pm 0.7 \%$ & $p<0.001$ \\
\hline Age & $72(52-89)$ & $65(34-88)$ & NS \\
\hline Body mass index $\left(\mathrm{kg} / \mathrm{m}^{2}\right)$ & $29.6 \pm 4.2$ & $28.6 \pm 4.2$ & NS \\
\hline Beta blockers (\% of patients/number of patients) & $90 \% / 19$ patients & $89 \% / 41$ patients & NS \\
\hline ACE inhibitors or AT1 blockers (\% of patients/number of patients) & $85 \% / 18$ patients & $67 \% / 31$ patients & NS \\
\hline Statins (\% of patients/number of patients) & $100 \% / 21$ patients & $96 \% / 44$ patients & NS \\
\hline Diuretics ( $\%$ of patients/number of patients) & $52 \% / 11$ patients & $31 \% / 14$ patients & NS \\
\hline Clopidogrel (\% of patients/number of patients) & $52 \% / 11$ patients & $48 \% / 22$ patients & NS \\
\hline Prasugrel (\% of patients/number of patients) & $48 \% / 10$ patients & $52 \% / 24$ patients & NS \\
\hline
\end{tabular}

TABLE 2: On-treatment platelet reactivity and prevalence of ADP receptor blocker nonresponders in T2D and ND patients.

\begin{tabular}{|c|c|c|c|}
\hline On-treatment platelet reactivity & T2D patients & ND patients & Significance \\
\hline \multicolumn{4}{|l|}{ LTA with ADP induction (\%) } \\
\hline Sample 1 & $57.2 \pm 26.4$ & $50.3 \pm 22.3$ & NS \\
\hline Sample 2 & $45.9 \pm 31.3$ & $37.0 \pm 20.8$ & NS \\
\hline \multicolumn{4}{|l|}{ PRI of VASP phosphorylation (\%) } \\
\hline Sample 1 & $59.4 \pm 30.9$ & $60.0 \pm 25.2$ & NS \\
\hline Sample 2 & $33.9 \pm 25.3$ & $38.6 \pm 29.3$ & NS \\
\hline ADP receptor blocker nonresponders (\% of patients/number of patients) & T2D patients & ND patients & Significance \\
\hline \multicolumn{4}{|l|}{ ADP receptor blocker nonresponders } \\
\hline Sample 1 & $52.4 \% / 11$ patients & $56.5 \% / 26$ patients & NS \\
\hline Sample 2 & $33.3 \% / 7$ patients & $30.4 \% / 14$ patients & NS \\
\hline
\end{tabular}

2.3. Statistical Analysis. Data were in the first step checked for normality with the Shapiro-Wilk test. Normally distributed continuous or interval-scaled variables are presented as mean \pm standard deviation (SD); otherwise median $(\mathrm{M})$ and quartile ranges from the lower quartile to the upper quartile were used. Group effects (i.e., differences between T2D and ND groups) were tested with $t$-test in the case of normally distributed data or with Mann-Whitney $U$ test when data distribution was asymmetrical. Differences between proportions (e.g., number of patients in T2D and ND groups) were tested with binominal tests. Categorical variables grouped in 2way contingency tables were analyzed using chi-square tests. The significance of $p<0.05$ was considered as a criterion for comparison between data sets with equal and unequal variances. The statistical analysis was performed with Statistica v. 7.0 (StatSoft Inc., Dell Software, Tulsa, Oklahoma, USA). Sample size calculation was based on the assumption of the incidence of HTPR among ADP receptor blockerstreated T2D patients reported in previously published studies $[9,10]$. The primary aim of this study was to clarify possible differences in ADP receptor blockers on-treatment platelet reactivity according to $\mathrm{T} 2 \mathrm{D}$ status. Choosing a two-sided value of 0.05 , we estimated that an overall sample size of 20 T2D patients and 20 control (nondiabetic) patients would be sufficient for statistical analysis. To reach even more valid sample, we decided to enroll more than 20 patients in each of the compared groups (however, we were able to enroll only one more T2D patient who met the inclusion criteria of the study). The statistical analysis was consulted with a professional and designed prior to patient enrollment to achieve a valid statistical evaluation of the results of the study.

\section{Results}

The time interval from ADP receptor blocker loading dose administration to the collection of sample 1 was $1.8 \pm 0.9$ hours and to the collection of sample 2 was $20.5 \pm 2.1$ hours. The mean platelet aggregability after the induction with ADP was $52.5 \pm 23.6 \%$ in sample 1 and $39.7 \pm 24.5 \%$ in sample 2. Examination of VASP phosphorylation showed mean PRI $59.7 \pm 26.9 \%$ in sample 1 and mean PRI $37.0 \pm 27.8 \%$ in sample 2 , respectively.

When comparing the T2D and ND group (Table 2) there were no significant differences in platelet aggregability after ADP neither in sample 1 nor in sample 2 (sample 1: $57.2 \pm$ $26.4 \%$ versus $50.3 \pm 22.3 \%$, NS; sample $2: 45.9 \pm 31.3 \%$ versus $37.0 \pm 20.8 \%$, NS). The PRI of VASP-P showed similar results: there were no significant differences between T2D and ND group neither in sample $1(59.4 \pm 30.9 \%$ versus $60.0 \pm 25.2 \%$, NS) nor in sample $2(33.9 \pm 25.3 \%$ versus $38.6 \pm 29.3 \%$, NS). The time interval from ADP receptor blocker loading dose administration to the sample collection was similar in T2D 
TABLE 3: Platelet aggregability and PRI of VASP-P in prasugrel- and clopidogrel-treated patients with acute STEMI (analysis of combined $\mathrm{T} 2 \mathrm{D}$ and ND patients and analysis of T2D patients, marked as T2D patients).

\begin{tabular}{lccc}
\hline LTA with ADP induction (\%) & Prasugrel-treated patients & Clopidogrel-treated patients & Significance \\
\hline Sample 1 & $45.9 \pm 26.2 \%$ & $59.2 \pm 18.8 \%$ & $p<0.05$ \\
Sample 2 & $24.9 \pm 17.7 \%$ & $51.7 \pm 22.8 \%$ & $<0.001$ \\
\hline LTA with ADP induction (\%) & Prasugrel-treated T2D patients & Clopidogrel-treated T2D patients & Significance \\
T2D patients & $43.3 \pm 20.6$ & $63.5 \pm 22.8$ & $p<0.05$ \\
\hline Sample 1 & $22.9 \pm 16.9$ & $60.3 \pm 26.6$ & $p<0.01$ \\
Sample 2 & Prasugrel-treated patients & Clopidogrel-treated patients & Significance \\
\hline PRI of VASP-P analysis (\%) & $52.7 \pm 29.7 \%$ & $68.9 \pm 19.9$ & $p<0.05$ \\
Sample 1 & $26.3 \pm 24.3 \%$ & $51.8 \pm 22.8 \%$ & $p<0.01$ \\
Sample 2 & Prasugrel-treated T2D patients & Clopidogrel-treated T2D patients & Significance \\
\hline PRI of VASP-P analysis (\%) & $46.8 \pm 33.5$ & $78.3 \pm 13.0$ & $p<0.05$ \\
T2D patients & $18.7 \pm 15.7$ & $56.8 \pm 18.7$ & $p<0.01$ \\
\hline Sample 1 & & & \\
Sample 2 & & &
\end{tabular}

and ND patients in both examinations (sample 1: $1.8 \pm 0.9$ hours versus $1.7 \pm 0.9$ hours; sample $2: 21.6 \pm 2.2$ hours versus $20.0 \pm 1.9$ hours).

Subsequently, an analysis of ADP receptor blocker nonresponders (Table 2) was performed. The difference in the prevalence of ADP receptor blocker nonresponders in T2D and ND patients did not reach statistical significance. However, the number of ADP receptor blocker nonresponders tended to be higher in T2D patients. In T2D patients an incomplete response on ADP receptor blocker (PRI > 50\%) was identified in $52.4 \%$ of patients in first sample and in $33.3 \%$ in second sample. While in ND group, $56.5 \%$ of patients were nonresponders in first sample and $30.4 \%$ of patients did not respond sufficiently in second sample. No significant differences in the prevalence of ADP blocker nonresponders between T2D prasugrel-treated and ND prasugrel-treated patients were found (sample 1: $40.0 \%$ versus $41.7 \%$, NS; sample $2: 10.0 \%$ versus $12.5 \%$, NS). In addition, the prevalence of ADP receptor blocker nonresponders did not differ significantly in clopidogrel-treated T2D patients compared to clopidogrel-treated ND patients (sample 1: 63.6\% versus 72.7\%, NS; sample 2: 54.6 versus 50.0\%, NS).

Prasugrel induced in this preliminary study significantly more potent platelet inhibition (Table 3 ) than that of clopidogrel. Prasugrel-treated patients had significantly lower residual platelet aggregation in both samples: $45.9 \pm 26.2 \%$ versus $59.2 \pm 18.8 \%(p<0.05)$ in sample 1 and $24.9 \pm 17.7 \%$ versus $51.7 \pm 22.8 \%(p<0.001)$ in sample 2 , respectively. Similarly, the PRI of VASP-P was significantly lower in prasugrel-treated patients in sample 1, as well as in sample 2 (sample 1: $52.7 \pm 29.7 \%$ versus $68.9 \pm 19.9, p<0.05$; sample 2: $26.3 \pm 24.3 \%$ versus $51.8 \pm 22.8 \%, p<0.01)$. Consistently, significantly lower residual platelet reactivity was found in prasugrel-treated T2D patients when compared to clopidogrel-treated T2D patients in both samples (sample $1: 43.3 \pm 20.6 \%$ versus $63.5 \pm 22.8 \%, p<0.05$; sample 2 : $22.9 \pm 16.9 \%$ versus $60.3 \pm 26.6 \%, p<0.01)$. Significantly better inhibition of ADP signaling pathway in prasugreltreated T2D patients was demonstrated with significantly lower PRI of VASP-P in both samples (sample 1: $46.8 \pm 33.5$ versus $78.3 \pm 13.0, p<0.05$; sample 2 : $18.7 \pm 15.7$ versus $56.8 \pm 18.7, p<0.01)$.

\section{Discussion}

T2D is a strong and independent risk factor of acute STEMI. T2D increases the risk of future complications in acute STEMI patients. Endothelial dysfunction [11], abnormalities in coagulation and fibrinolysis [12], and platelet dysfunction $[6,7]$ are important factors of worsen prognosis of acute STEMI in T2D patients. However, several studies pointed at a failure in antiplatelet response to clopidogrel which might be connected with insulin resistance and T2D [8-10]. These studies consistently identified higher residual on-treatment platelet reactivity in clopidogrel-treated T2D patients and higher number of clopidogrel nonresponders among diabetic patients. Moreover, HTPR was in these studies consistently connected with higher risk of adverse ischemic events after PCI. The mechanism of this phenomenon remains unclear. The study performed by Erlinge et al. [9] showed that platelets of diabetic patients with clopidogrel HTPR responded well to ex vivo administration of clopidogrel active metabolite. This finding suggests a potential interaction between T2D and pharmacokinetic processes of clopidogrel metabolism.

On the other hand, the results of this preliminary prospective observation did not confirm the significantly higher residual platelet reactivity or significantly higher prevalence of HTPR in T2D acute STEMI patients undergoing pPCI of culprit coronary lesion. The exact explanation of this observation is recently missing. The time interval from drug administration to blood sample collection did not differ significantly in T2D and ND patients neither in sample 1 nor in sample 2. The differences in time interval from drug 
dosing to blood sampling therefore cannot explain the results obtained in this study.

Another possible explanation may be the fact that the majority of studies pointing on the higher prevalence of HTPR among T2D patients were performed on a sample of stable coronary/ischemic heart disease patients. It is generally accepted that patients with acute STEMI represent a special group with different clinical and risk profile. It is therefore possible that results obtained from the studies on stable patients might not be fully applicable in high risk ACS patients. However, study performed by Cuisset et al. identified high prevalence of clopidogrel nonresponders (50\% of patients) also in T2D patients undergoing PCI for ACS [13]. In the light of these data, the real influence of acute coronary syndrome itself on the prevalence of HTPR in T2D patients seems not to be probable.

An alternative explanation of similar on-treatment platelet reactivity in T2D and ND patients in this study might be a possibility that the failure in antiplatelet response is in T2D patients specifically associated with clopidogrel. The administration of newer ADP receptor blockers might not be connected with such a failure in on-treatment response [14]. In fact, prasugrel administration in the TRITON-TIMI 38 trial achieved the most pronounced clinical benefit just in T2D patients [15]. Prasugrel was in our preliminary study quite frequently used among T2D (48\% of patients), as well as among ND patients (52\% of patients). Prasugrel induced in our study significantly more potent platelet inhibition than that of clopidogrel in both samples. The efficacy of newer ADP receptor blockers among high risk T2D acute coronary syndrome patients was consistently proven in a recent single centre randomized clinical study performed by Laine et al. [16]. It is therefore possible that more frequent use of newer ADP receptor blockers (such as prasugrel) would resolve the insufficient platelet inhibition, which might be seen in clopidogrel-treated T2D patients. Nevertheless, the relationship between T2D and ADP receptor blocker ontreatment platelet reactivity remains inadequately explained [17-19] and further studies would be needed for the final clarification of this issue.

\section{Limitations}

There were some important limitations of our analysis. First, this study had a prospective observational design and not a randomized double blinded one. The decision on ADP receptor blocker therapy strategy (clopidogrel versus prasugrel) was left to the physician who performed the diagnostic ECG record (general practitioner, cardiologist, ED physician, etc.). Therefore the data obtained in this study do not have the evidence power of data from a randomized double blinded trial. Second, a low sample size might be a limitation of the study. A relatively small patient sample cannot guarantee significant power. Third, a relatively short time interval from ADPRB loading dosing to first sampling (especially in the settings of acute coronary syndrome) might be another limitation of this study. It is already known that time to peak platelet inhibition is generally prolonged in acute STEMI patients and also in stable coronary artery disease patients with T2D [19]. However, in this study, we wanted to test the impact of T2D on the on-treatment response in previously ADPRB naive acute STEMI patients undergoing pPCI. Therefore we decided to test the antiplatelet response prior to $\mathrm{pPCI}$ and one day after the procedure rather than after a prolonged ADPRB administration. Nevertheless, this fact could affect the ontreatment platelet reactivity detected in our study. Finally, the drug compliance was not proven by a laboratory testing (measurement of clopidogrel metabolite, etc.). Although, the drug compliance was in this study carefully monitored by a healthcare professional who supervised all antiplatelet drug administrations, the exact confirmation of drug compliance with laboratory assessment is missing. The results of this study should be confirmed in large, similarly designed, randomized trial.

\section{Conclusion}

This prospective study did not confirm the higher residual platelet reactivity and higher prevalence of HTPR in T2D acute STEMI patients undergoing pPCI of culprit coronary lesion. However, the results of this study are preliminary and further studies on larger sample sizes would be definitely needed for the final clarification of this issue.

\author{
Abbreviations \\ T2D: $\quad$ Type 2 diabetes \\ STEMI: ST elevation myocardial infarction \\ ADP: $\quad$ Adenosine diphosphate \\ VASP-P: Vasodilator-stimulated phosphoprotein \\ phosphorylation \\ pPCI: Primary percutaneous coronary \\ intervention \\ ND: Nondiabetic \\ ACS: Acute coronary syndrome \\ HTPR: High on-treatment platelet reactivity \\ HbA1C: Hemoglobin AlC \\ DCCT: Diabetes Control and Complications Trial \\ LTA: Light transmission aggregometry \\ PGE1: Prostaglandin E1 \\ MFIc: Corrected mean fluorescence intensity \\ PRI: Platelet reactivity index \\ SD: $\quad$ Standard deviation \\ ECG: Electrocardiography \\ ED: Emergency department.
}

\section{Ethical Approval}

This research was done according to ethical standards and was approved by the local ethical committee.

\section{Consent}

The patients agreed to participate in the research and signed written informed consent for study participation. 


\section{Competing Interests}

The authors have no conflict of interests to declare.

\section{Acknowledgments}

This study was supported by project APVV (Slovak Research and Development Agency) 0222-11 and by research project of Slovak Society of Cardiology 2012-2015. The authors would like to thank Dr. Michal Bugala, Ph.D. (Technical University in Zvolen), for performing the statistical analysis.

\section{References}

[1] P. W. F. Wilson, R. B. D’Agostino, D. Levy, A. M. Belanger, H. Silbershatz, and W. B. Kannel, "Prediction of coronary heart disease using risk factor categories," Circulation, vol. 97, no. 18, pp. 1837-1847, 1998.

[2] P. W. F. Wilson, "Diabetes mellitus and coronary heart disease," American Journal of Kidney Diseases, vol. 32, no. 5, supplement 3, pp. S89-S100, 1998.

[3] H. C. McGill and C. A. McMahan, "Determinants of atherosclerosis in the young. Pathobiological Determinants of Atherosclerosis in Youth (PDAY) research group," The American Journal of Cardiology, vol. 82, pp. 30-36, 1998.

[4] D. R. Holmes Jr., P. B. Berger, J. S. Hochman et al., "Cardiogenic shock in patients with acute ischemic syndromes with and without ST-segment elevation," Circulation, vol. 100, no. 20, pp. 2067-2073, 1999.

[5] G. Zuanetti, R. Latini, A. P. Maggioni, L. Santoro, Maria Grazia Franzosi, and GISSI-2 Investigators, "Influence of diabetes on mortality in acute myocardial infarction: data from the GISSI-2 study," Journal of the American College of Cardiology, vol. 22, no. 7, pp. 1788-1794, 1993.

[6] D. Aronson, Z. Bloomgarden, and E. J. Rayfield, "Potential mechanisms promoting restenosis in diabetic patients," Journal of the American College of Cardiology, vol. 27, no. 3, pp. 528-535, 1996.

[7] D. Tschoepe, P. Roesen, L. Kaufmann et al., "Evidence for abnormal platelet glycoprotein expression in diabetes mellitus," European Journal of Clinical Investigation, vol. 20, no. 2, pp. 166170, 1990.

[8] M. Samoš, R. Šimonová, F. Kovář et al., "Clopidogrel resistance in diabetic patient with acute myocardial infarction due to stent thrombosis," The American Journal of Emergency Medicine, vol. 32, no. 5, pp. 461-465, 2014.

[9] D. Erlinge, C. Varenhorst, O. Ö. Braun et al., "Patients with poor responsiveness to thienopyridine treatment or with diabetes have lower levels of circulating active metabolite, but their platelets respond normally to active metabolite added ex vivo," Journal of the American College of Cardiology, vol. 52, no. 24, pp. 1968-1977, 2008.

[10] D. J. Angiolillo, E. Bernardo, M. Sabaté et al., "Impact of platelet reactivity on cardiovascular outcomes in patients with type 2 diabetes mellitus and coronary artery disease," Journal of the American College of Cardiology, vol. 50, no. 16, pp. 1541-1547, 2007.

[11] P. Kubisz, P. Chudý, J. Staško et al., "Circulating vascular endothelial growth factor in the normo- and/or microalbuminuric patients with type 2 diabetes mellitus," Acta Diabetologica, vol. 47 , no. 2, pp. 119-124, 2010.
[12] P. Chudý, D. Kotuličová, J. Staško, and P. Kubisz, “The relationship among TAFI, t-PA, PAI- 1 and F1 +2 in type 2 diabetic patients with normoalbuminuria and microalbuminuria," Blood Coagulation \& Fibrinolysis, vol. 22, no. 6, pp. 493-498, 2011.

[13] T. Cuisset, B. Gaborit, N. Dubois et al., "Platelet reactivity in diabetic patients undergoing coronary stenting for acute coronary syndrome treated with clopidogrel loading dose followed by prasugrel maintenance therapy," International Journal of Cardiology, vol. 168, no. 1, pp. 523-528, 2013.

[14] M. Fedor, M. Samoš, R. Šimonová et al., "Monitoring the efficacy of ADP inhibitor treatment in patients with acute STEMI post-PCI by VASP-P flow cytometry assay," Clinical and Applied Thrombosis/Hemostasis, vol. 21, no. 4, pp. 334-338, 2015.

[15] S. Wiviott, E. Braunwald, C. McCabe et al., "Prasugrel versus clopidogrel in patients with acute coronary syndromes," The New England Journal of Medicine, vol. 357, pp. 2001-2015, 2007.

[16] M. Laine, C. Frère, R. Toesca et al., “Ticagrelor versus prasugrel in diabetic patients with an acute coronary syndrome. A pharmacodynamic randomised study," Thrombosis and Haemostasis, vol. 111, no. 2, pp. 273-278, 2013.

[17] S. I. Al-Azzam, K. H. Alzoubi, O. F. Khabour et al., "Factors that contribute to clopidogrel resistance in cardiovascular disease patients: environmental and genetic approach," International Journal of Clinical Pharmacology and Therapeutics, vol. 51, no. 3, pp. 179-186, 2013.

[18] H. M. Hall, S. Banerjee, and D. K. McGuire, "Variability of clopidogrel response in patients with type 2 diabetes mellitus," Diabetes and Vascular Disease Research, vol. 8, no. 4, pp. 245253, 2011.

[19] D. J. Angiolillo, J. A. Jakubowski, J. L. Ferreiro et al., "Impaired responsiveness to the platelet $\mathrm{P} 2 \mathrm{Y}_{12}$ receptor antagonist clopidogrel in patients with type 2 diabetes and coronary artery disease," Journal of the American College of Cardiology, vol. 64, no. 10, pp. 1005-1014, 2014. 


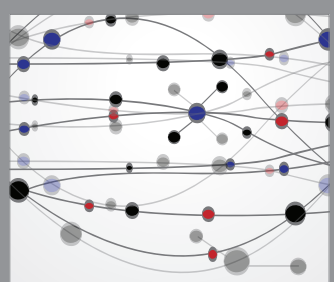

The Scientific World Journal
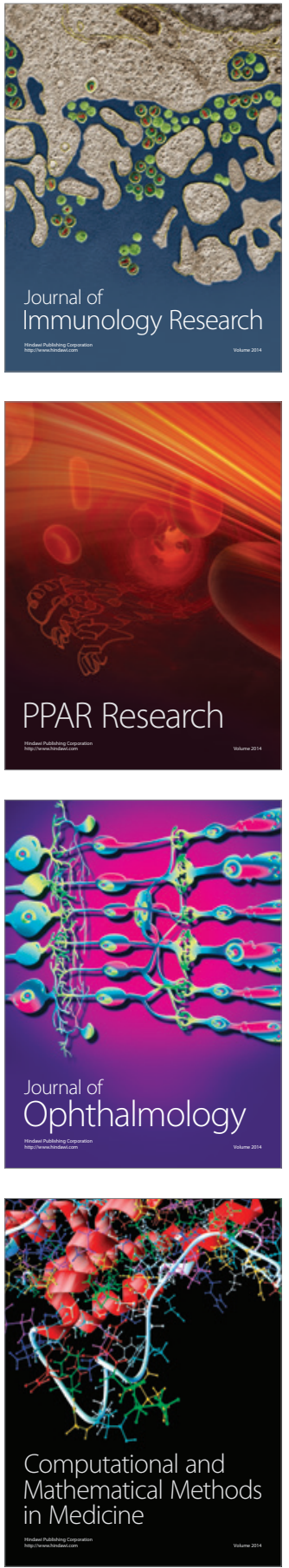

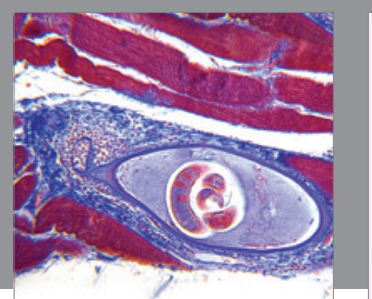

Gastroenterology Research and Practice

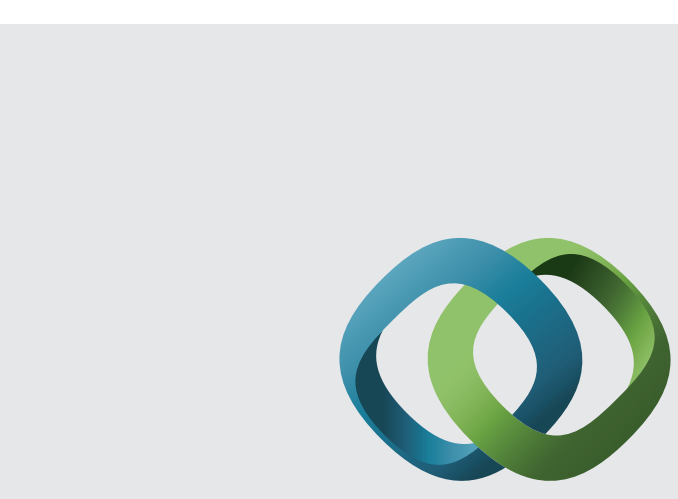

\section{Hindawi}

Submit your manuscripts at

http://www.hindawi.com
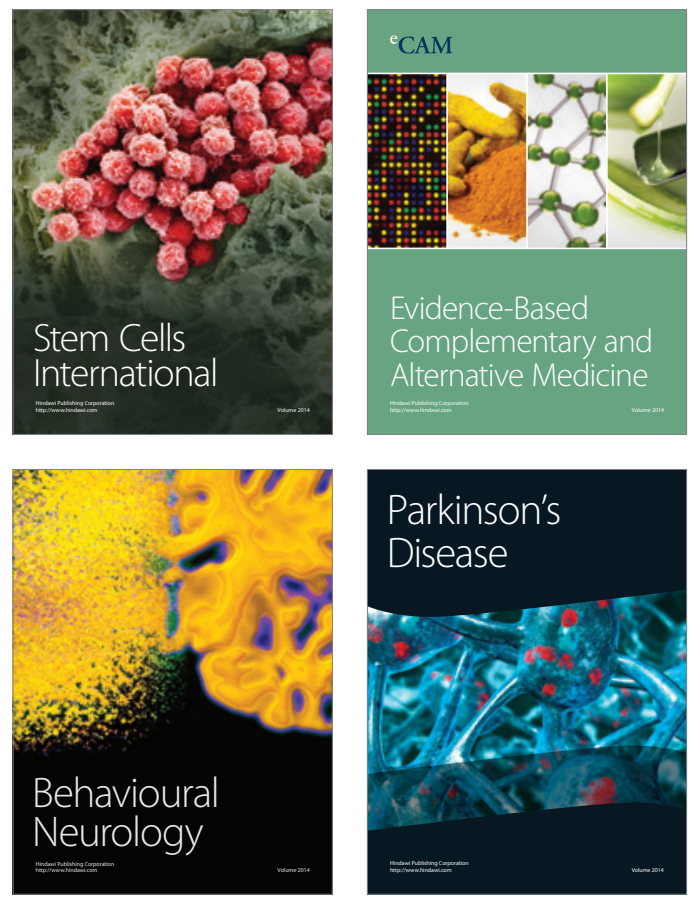
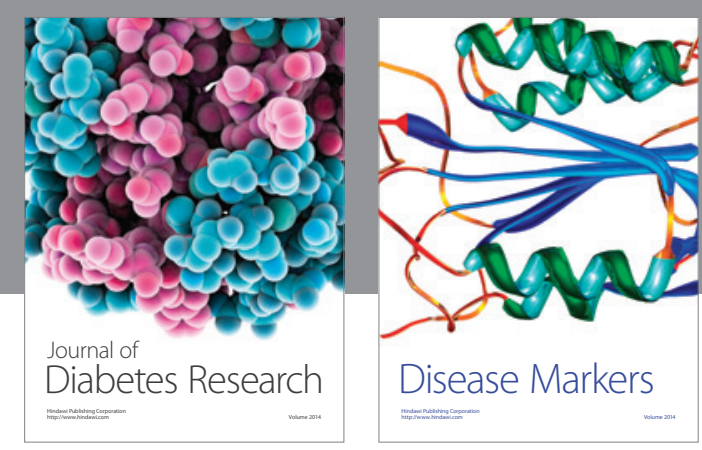

Disease Markers
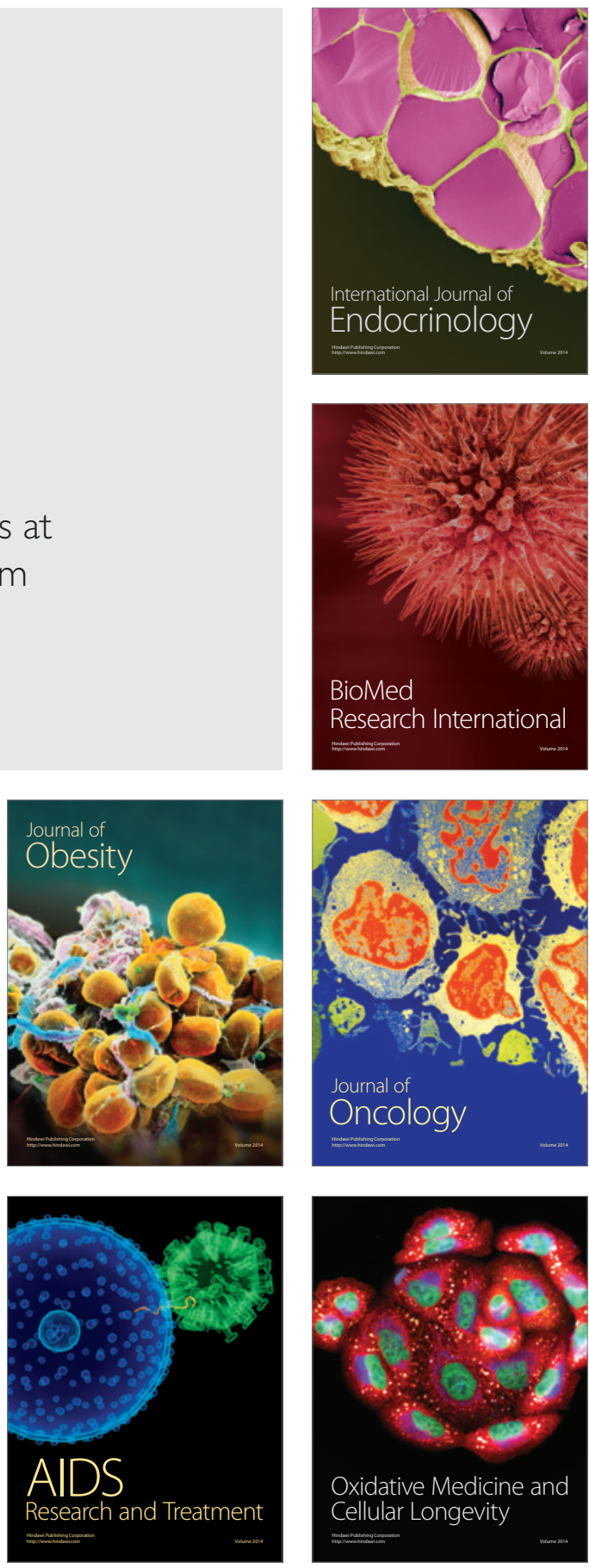